\title{
Model-Based Fatique Fractographs Texture Analysis
}

\author{
Michal Haindl ${ }^{1}$ and Hynek Lauschmann ${ }^{2}$ \\ 1 Institute of Information Theory and Automation, Academy of Sciences CR \\ Prague, CZ182 08, Czech Republic \\ haindl@utia.cas.cz \\ 2 Faculty of Nuclear Science and Physical Engineering, Czech Technical University \\ Prague, CZ120 00, Czech Republic \\ hynek. lauschmannl@fjfi.cvut.cz
}

\begin{abstract}
A novel model-based approach for estimation of the velocity of crack growth from microfractographical images is proposed. These images are represented by a Gaussian Markov random field model and the crack growth rate is modelled by a linear regression model in the Gaussian-Markov parameter space. The method is numerically very efficient because both crack growth rate model parameters as well as the underlying random field model parameters are estimated using fast analytical estimators.
\end{abstract}

\section{Introduction}

The quantitative microfractography of fatigue failures is concerned mainly with the investigation of the history of a fatigue crack growth process. Specimens of the material are loaded under service conditions and the crack growth process is recorded. Fracture surfaces images produced by a scanning electron microscope (SEM) are studied to relate image morphological information of the crack surface with the macroscopic crack growth rate (CGR). The crack growth process is reconstituted using integration of CGR along the crack growth direction.

Traditional fractographical methods are based on strictly defined fractographic features measurable in the morphology of a fracture surface. In the case of fatigue analysis, these features are striations [12,13], i.e., fine parallel grooves in the fracture surface. However such methods cannot be used when striations are partially occluded, typically due to corrosion. For such cases, a family of methods is being developed called textural fractography [14]-[22]. The proposed method estimates CGR from textural features derived from a Markovian underlying model.

For the application of the textural method, especially suitable is the mezoscopic dimensional area with SEM magnifications between macro- and microfractography (about 30-500×). These magnifications were traditionally seldom used in the past due to the absence of measurable objects in corresponding images (for example see Fig.1). Setting the magnification is limited by several conditions related to individual images, to the whole set of images and to image 

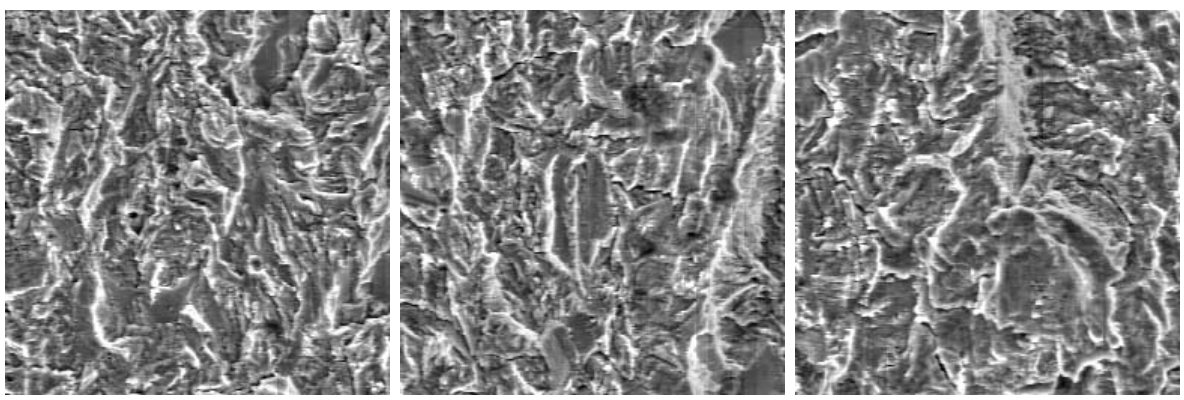

Fig. 1. Cuttings $(256 \times 256)$ from small, medium and great CGR

discretization. Images require preprocessing to eliminate lighting variations to get homogeneous image set for subsequent textural analysis. Fractographic information is extracted in the form of integral parameters of the whole image. Such parameters might be different statistical (e.g., correlations, statistical moments, etc.) or textural features.

In the presented method, the fatique image is assumed to be described by a Markov random field (MRF) fitted to this image. Although the MRF models generally suffer with time consuming iterative methods for both parameter estimation as well as for the synthesis, the Gaussian Markov random field (GMRF) model used in this paper belongs to few exceptional Markovian models which avoid the time consuming Markov chain Monte Carlo simulations so typical for the rest of Markovian models family.

Modelling monospectral still images require two dimensional models. Among such possible models the Gaussian Markov random fields are appropriate for fractographical texture modelling not only because they do not suffer with some problems (e.g., sacrificing considerable amount of image information, nonlinear parameter estimation, etc.) of alternative options (see [3,4,5,6,10,11] for details) but they are also easy to synthesize and still flexible enough to imitate a large set of fractographical textures. While the random field based models quite successfully represent high frequencies present in natural fatique textures low frequencies are much more difficult for them. However, the model does not need to generate a realistic fatique texture. For the crack velocity estimation it is sufficient to produce discriminative features.

\section{Fatique Images Model}

Single monospectral SEM images are assumed to be independently modelled by their dedicated Gaussian Markov random field models (GMRF) as follows. The Markov random field (MRF) is a family of random variables with a joint probability density on the set of all possible realisations $Y$ indexed (in our application) on a finite two-dimensional rectangular $(N \times M)$ lattice $I$, subject to following 
conditions:

$$
p(Y)>0
$$

and

$$
p\left(Y_{r} \mid Y_{s} \forall s \in I \backslash\{r\}\right)=p\left(Y_{r} \mid Y_{s} \forall s \in I_{r}\right),
$$

where $r=\left\{r_{1}, r_{2}\right\}$ is the multiindex with the row and column indices, respectively. $I_{r} \subset I$ is a $2 \mathrm{D}$ symmetric contextual support set of the monospectral random field.

If the local conditional density of the MRF model (3) is Gaussian, we obtain the continuous Gaussian Markov random field model (GMRF):

$$
p\left(Y_{r} \mid Y_{s} \forall s \in I_{r}\right)=\left(2 \pi \sigma^{2}\right)^{-\frac{1}{2}} \exp \left\{-\frac{1}{2 \sigma^{2}}\left(Y_{r}-\tilde{\mu}_{r}\right)^{2}\right\}
$$

where the mean value is

$$
\begin{aligned}
E\left\{Y_{r} \mid Y_{s} \forall s \in I \backslash\{r\}\right\} & =\tilde{\mu}_{r} \\
& =\mu_{r}+\sum_{s \in I_{r}} a_{s}\left(Y_{r-s}-\mu_{r-s}\right)
\end{aligned}
$$

and $\sigma, a_{s} \forall s \in I_{r}$ are unknown parameters. The 2D GMRF model can be also expressed [3] as a stationary non-causal correlated noise driven 2D autoregressive process:

$$
\tilde{Y}_{r}=\sum_{s \in I_{r}} a_{s} \tilde{Y}_{r-s}+e_{r}
$$

where $\tilde{Y}_{r}=Y_{r}-\mu_{r}$ are centered variables, the noise $e_{r}$ are random variables with zero mean

$$
E\left\{e_{r}\right\}=0
$$

The $e_{r}$ noise variables are mutually correlated

$$
\begin{aligned}
R_{e} & =E\left\{e_{r} e_{r-s}\right\} \\
& = \begin{cases}\sigma^{2} & \text { if } s=(0,0), \\
-\sigma^{2} a_{s} & \text { if } s \in I_{r}, \\
0 & \text { otherwise }\end{cases}
\end{aligned}
$$

Correlation functions have the symmetry property

$$
E\left\{e_{r} e_{r+s}\right\}=E\left\{e_{r} e_{r-s}\right\}
$$

hence the neighbourhood support set and their associated coefficients have to be symmetric, i.e.,

$$
s \in I_{r} \Rightarrow-s \in I_{r}
$$

and $a_{s}=a_{-s}$. 


\subsection{Parameter Estimation}

The selection of an appropriate GMRF model support is important to obtain good results in modelling of a given random field. If the contextual neighbourhood is too small it can not capture all details of the random field. Inclusion of the unnecessary neighbours on the other hand add to the computational burden and can potentially degrade the performance of the model as an additional source of noise. We use the hierarchical neighbourhood system $I_{r}$, e.g., the first-order neighbourhood is $I_{r}^{1}=\{r-(0,1), r+(0,1), r-(1,0), r+(1,0)\}$,

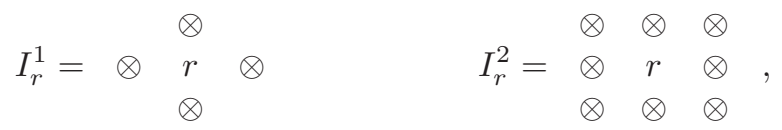

etc. An optimal neighbourhood is detected using the correlation method [7] favouring neighbours locations corresponding to large correlations over those with small correlations.

Parameter estimation of a MRF model is complicated by the difficulty associated with computing the normalization constant. Fortunately the GMRF model is an exception where the normalization constant is easy to obtain. However either Bayesian or ML estimate requires iterative minimization of a nonlinear function. Therefore we use the pseudo-likelihood estimator which is computationally simple although not efficient. The pseudo-likelihood estimate for $a_{s}$ parameters has the form

$$
\begin{aligned}
\hat{\gamma}^{T} & =\left[\begin{array}{ll}
\hat{a}_{s} & \forall s \in I_{r}
\end{array}\right]^{T} \\
& =\left[\sum_{r \in I} X_{r} X_{r}^{T}\right]^{-1} \sum_{r \in I} X_{r} \tilde{Y}_{r}
\end{aligned}
$$

where

$$
X_{r}=\left[\tilde{Y}_{r-s} \quad \forall s \in I_{r}\right]^{T}
$$

and

$$
\hat{\sigma}^{2}=\frac{1}{M N} \sum_{r=1}^{M N}\left(\tilde{Y}_{r}-\hat{\gamma} X_{r}\right)^{2} .
$$

Alternatively this estimator can be computed recursively $[8,9]$.

\subsection{Crack Growth Rate Model}

We assume that the crack growth rate $v(i)$ is linearly dependent on GMRF parameters describing corresponding fatique images, i.e.,

$$
v(i)=\sum_{j=1}^{\nu} b_{j} a_{j, i}+\epsilon_{i}=\theta \gamma_{i}^{T}+\epsilon_{i}, \quad i=1, \ldots, n
$$



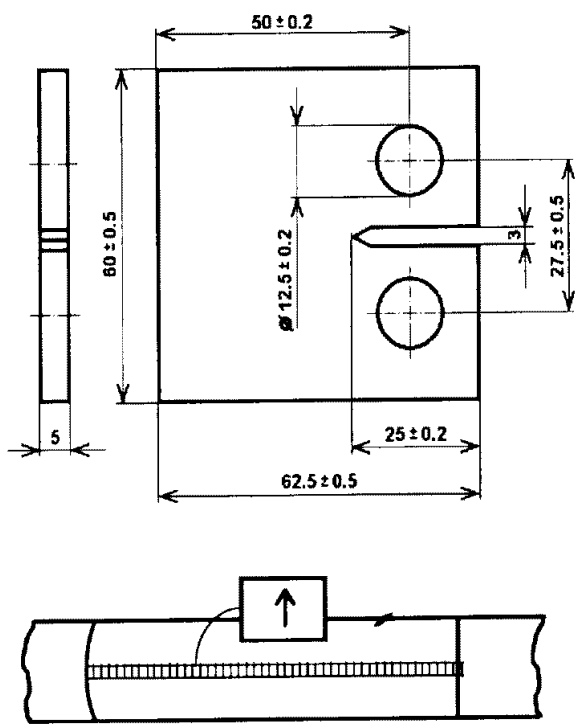

fatigue

Fig. 2. The fatique test specimen and the location of images in the fatique crack surface

where $b_{s}$ are unknown parameters, $\theta=\left[b_{1}, \ldots, b_{\nu}\right]$,

$\nu=\operatorname{card}\left\{I_{r}\right\}+1$

$n$ is the number of fatique images and $a_{s}$ are RF pseudo-likelihood estimates (7),(9). The growth rate is assumed to have independent Gaussian measurement error $\epsilon_{i}$ with standard deviation $\eta_{j} j=1, \ldots, \nu$. We can assume an overestimated set of equations, i.e., $n \gg \nu$ hence the $b_{s}$ parameters can be for example estimated using the least square estimator

$$
\hat{\theta}^{T}=\left(\Gamma^{T} \Gamma\right)^{-1} \Gamma^{T} V
$$

where

$$
V=[v(1), \ldots, v(n)]^{T}
$$

and

$$
\Gamma=\left[\gamma_{1}^{T}, \ldots, \gamma_{n}^{T}\right]^{T}
$$

is a $n \times \nu$ design matrix. Finally the velocity estimator is

$$
\hat{v}(i)=\hat{\theta} \gamma_{i}^{T}
$$

The alternative option is a Bayesian estimator for both - unknown parameters from $\theta$ as well as for the optimal model selection (i.e., selection of an optimal subset of the variables $\left.a_{j, i}\right)$. 


\section{Results}

The method was applied on data from four fatigue experiments with specimens from stainless steel AISI 304L used in nuclear power plants. The specimen type was CT (Fig.2) with the initial notch length $12.5 \mathrm{~mm}$. Constant cycle loading with parameters $\triangle F=3400 \mathrm{~N}, R=0.3, f=1 \mathrm{~Hz}$ occured in water at $20^{\circ} \mathrm{C}$. The crack length was measured by COD. Fatigue crack surfaces were documented using SEM with magnification $200 \times$. The sequence of images was located in the middle of the crack surface (Fig.2) and the images were distanced by $0.4 \mathrm{~mm}$. The direction of the crack growth in images is bottom-up. The real area of one image is about $0.6 \times 0.45 \mathrm{~mm}$ (the images overlap by $0.05 \mathrm{~mm}$ ). The whole experimental set contains 164 images. Fig.1 shows examples of typical textures - cuttings $256 \times 256$ pixels from normalized images (size $1200 \times 1600$ pixels).

The estimation quality was evaluated using the mean absolute error:

$$
\zeta=\frac{1}{n} \sum_{i=1}^{n}|v(i)-\hat{v}(i)|
$$

and the overall velocity estimation error

$$
\varsigma=\frac{100 \zeta}{\bar{v}}
$$

where $\bar{v}$ is the average velocity in the measurements set. These textures were modelled using the fifth order GMRF model. Although all modelled textures are non stationary and thus violate the GMRF model assumption, the crack rate estimates are fairly accurate. The rate estimates can be further improved if we select a subset of variables $\gamma_{i}$ for the model for example by eliminating variables with low correlation with velocity.

\section{Conclusions}

Our test results of the algorithm on stainless steel fatigue images are encouraging. Some estimated crack rates match true velocities within measurement accuracy. Overall velocity estimation error was $\varsigma=30 \%$ but further improvement is possible if we increase the GMRF model order or introduce a multiresolution MRF model. The proposed method allows quantitative estimation of the crack growth rate while it has still moderate computation complexity. The method does not need any time-consuming numerical optimization like for example some Markov chain Monte Carlo method.

\section{Acknowledgements}

This research was supported by the Grant Agency of the Czech Republic under Grants 102/00/0030 and 106/00/1715. 


\section{References}

1. Bennett, J., Khotanzad, A.: Multispectral random field models for synthesis and analysis of color images. IEEE Trans. on Pattern Analysis and Machine Intelligence 20 (1998) 327-332

2. Bennett, J., Khotanzad, A.: Maximum likelihood estimation methods for multispectral random field image models. IEEE Trans. on Pattern Analysis and Machine Intelligence 21 (1999) 537-543

3. Besag, J.: Spatial interaction and the statistical analysis of lattice systems. Journal of the Royal Statistical Society, Series B 36 (1974) 192-236 843, 844

4. Gagalowicz, A., Ma, S., Tournier-Laserve, C.: Efficient models for color textures. In: Proceedings of Int. Conf. Pattern Recognition, IEEE, Paris, (1986) 412-414 843

5. Haindl, M.: Texture synthesis. CWI Quarterly 4 (1991) 305-331 843

6. Haindl, M.: Texture modelling. In: Proceedings of the World Multiconference on Systemics, Cybernetics and Informatics Int. Inst. of Informatics and Systemics, Orlando, (2000) 634-639 843

7. Haindl, M., Havlíček, V.: Multiresolution colour texture synthesis. In: Proceedings of the 7th International Workshop on Robotics in Alpe-A dria-Danube Region, ASCO Art, Bratislava, (1998) 297-302 845

8. Haindl, M.: Texture Segmentation Using Recursive Markov Random Field Parameter Estimation. In: Proceedings of the 11th Scandinavian Conference on Image Analysis, Pattern Recognition Society of Denmark, Lyngby, (1999) 771-776 845

9. Haindl, M.: Recursive Square-Root Filters. In: Proceedings of the 15th IAPR Int. Conf. on Pattern Recognition II, IEEE Press, Los Alamitos, (2000) 1018-1021 845

10. Kashyap, R. L.: Analysis and Synthesis of Image Patterns by Spatial Interaction Models, In: Progress in Pattern Recognition 1, Elsevier, North-Holland, (1981) $43-50 \quad 843$

11. Kashyap, R. L., Eom, K.: Estimation in long-memory time series model. J. of Time Series Anal. 9 (1988) 35-41 843

12. Lauschmann, H.: I Computer Aided Fractography: The automatical evaluation of striation parameters. Engineering Mechanics 5 (1998) 377-380 842

13. Lauschmann H.: Textural fractography: estimation of the mean striation spacing and direction. In: Int. Conf. on Stereology and Image Analysis in Materials Science, Polish Society for Stereology, Cracow, (2000) 241-246 842

14. Lauschmann H.: Computer aided fractography. In: Proceedings of international conference Fractography 97, Institute of Materials Research of the Slovak Academy of Sciences, KoÜice, (1997) 181-188 842

15. Lauschmann H., Benes V.: Spatial statistics in material research. In: Industrial statistics, Physica-Verlag, Hiedelberg, (1997) 285-293

16. Cejka V., Benes V.: Computer Aided Fractography: Methods for evaluation of image anisotropy. In: Proceedings Int. Conf. on Stereology, Spatial Statistics and Stochastic Geometry, Union of Czech Mathematicians and Physicists, Prague, (1999) 89-94

17. Lauschmann H.: Computer Aided Fractography: The spectral analysis of fatigue crack images. In: Int. Conf. on Stereology, Spatial Statistics and Stochastic Geometry, Union of Czech Mathematicians and Physicists, Prague, (1999) 171-176

18. Lauschmann H.: Textural analysis of fatigue crack surfaces - Image pre-processing. Acta Polytechnica 40 (2000) 123-129 
19. Lauschmann H., Adamek J., Nedbal I. Textural fractography: Spectral analysis of images of fatigue crack surfaces. In: Fractography 2000, Institute of Materials Research of the Slovak Academy of Sciences, KoÜice (2000) 313-320

20. Lauschmann H.: A database-oriented analysis of a fibre process in fractography. Image Analysis and Stereology (Suppl.1) 20 (2001) 379-385

21. Lauschmann H., Racek O.: Textural fractography: Application of Gibbs random fields. In: Proc. 3rd Int. Conf. Materials Structure \& Micromechanics of Fracture, University of Technology, Brno, (2001)

22. Lauschmann H., Tùma M., Racek O., Nedbal I.: Textural fractography. Image Analysis and Stereology (Suppl.1) 20 (2001) 842 